2. Atik E, Barbero-Marcial M, Tanamati C, Kajita L, Ebaid M, Jatene A. Anomalous origin of the left coronary artery from the right pulmonary artery with intramural aortic trajectory. Clinicosurgical diagnostic implications. Arq Bras Cardiol. 1999; 73:181-90.

3. Turley K, Szarnicki RJ, Flachsbart KD, Richter RC, Popper RW, Tarnoff H. Aortic implantation is possible in all cases of anomalous origin of the left coronary artery from the pulmonary artery. Ann Thorac Surg. 1995;60:84-9.

4. Goldberg SP, Mitchell MB, Campbell DN, Tissot C, Lacour-Gayet F. Anomalous left coronary artery from the pulmonary artery with an intramural course within the aortic wall: report of 3 surgical cases. J Thorac Cardiovasc Surg. 2008;135: 696-8.

5. Adachi I, Kagisaki K, Yagihara T, Hagino I, Ishizaka T, Kobayashi J, et al. Unroofing aortic intramural left coronary artery arising from right pulmonary artery. Ann Thorac Surg. 2008;85:675-7.

6. Barbero-Marcial M, Tanamati C, Atik E, Ebaid M, Jatene A. Anomalous origin of the left coronary artery from the pulmonary artery with intramural aortic route: diagnosis and surgical treatment. J Thorac Cardiovasc Surg. 1999;117: 823-5.

\title{
Placement of 2 implantable centrifugal pumps to serve as a total artificial heart after cardiectomy
}

\author{
Martin Strueber, MD, Jan D. Schmitto, MD, Ingo Kutschka, MD, and Axel Haverich, MD, Hannover, \\ Germany
}

In recent years, the role of ventricular assist devices for management of chronic heart failure has continued to increase. This increase stems mainly from the success of improved technology, greater surgical experience, and increasing experience with management of ventricular assist device-related complications. Collectively, these advances have led to overall better outcomes.

The HeartWare Ventricular Assist Device (HVAD; HeartWare International, Inc, Framingham, Mass) has become an established mechanical support therapy for left ventricular failure. ${ }^{1,2}$ Moreover, the use of the HVAD for biventricular support has been successfully reported. ${ }^{3,4}$

We report the case of a 51-year-old man presenting with a ventricular septal defect after myocardial infarction, not amenable to surgical repair. Two centrifugal blood pumps were then implanted after cardiectomy, serving as a total artificial heart intended to be used as a bridge-to-transplant device.

\section{CLINICAL SUMMARY}

The patient was a 51-year-old man who initially came to a regional hospital with acute coronary syndrome. The

\footnotetext{
From the Department of Cardiothoracic, Transplantation and Vascular Surgery, Hannover Medical School, Hannover, Germany.

Disclosures: Authors have nothing to disclose with regard to commercial support. Received for publication May 12, 2011; revisions received July 7, 2011; accepted for publication July 19, 2011; available ahead of print Aug 19, 2011.

Address for reprints: Martin Strueber, MD, Professor of Thoracic Transplantation, Director of the Mechanical Cardiac Circulatory Support Program, Department of Cardiothoracic, Transplantation and Vascular Surgery, Hannover Medical School, Carl-Neuberg Strasse 1, 30625 Hannover, Germany (E-mail: strueber.martin@ mh-hannover.de).

J Thorac Cardiovasc Surg 2012;143:507-9

$0022-5223 / \$ 36.00$

Copyright (C) 2012 by The American Association for Thoracic Surgery doi:10.1016/j.jtcvs.2011.07.034
}

cardiac catheterization performed showed complete occlusion of the right coronary artery proximally, resulting in a large posterolateral and septal myocardial infarction. On echocardiography, biventricular failure and a large ventricular septal defect were seen. Despite maximal inotropic support, a Lifebridge extracorporeal support system (LIFEBRIDGE Medizintechnik AG, Ampfing, Germany) was implanted for hemodynamic stability. The patient was transferred by helicopter on an emergency basis to our institution in Hannover, Germany.

Transesophageal echocardiography was repeated, confirming biventricular failure, a large ventricular septal defect, and new-onset aortic regurgitation. The aortic regurgitation limited the effectiveness of the life support system, thereby mandating surgical intervention.

Intraoperatively, after median sternotomy the patient was switched from the life-support system to a conventional heart-lung machine with transfemoral venous cannulation and arterial cannulation of the ascending aorta. Initially, aortic valve replacement with a biologic prosthesis was performed. On visual inspection after ventriculotomy, however, a massive posterior infarction with destruction of the posterolateral wall was seen. This included the entire ventricular septum and anterior papillary muscle, far beyond conventional surgical repair.

The only option was the implantation of a total artificial heart. Under complete cardiopulmonary bypass, the aortic valve prosthesis, the pulmonary valve, and both ventricles were resected so as to preserve the atria and the annuli of both atrioventricular valves. An epicardial HVAD sewing ring was implanted on the ventricular aspect of the mitral annulus with a 2-0 running Prolene suture (Ethicon, Inc, Somerville, NJ). The suture was supported by felt strips running outside the annulus around the left atrium. The sewing ring and the 

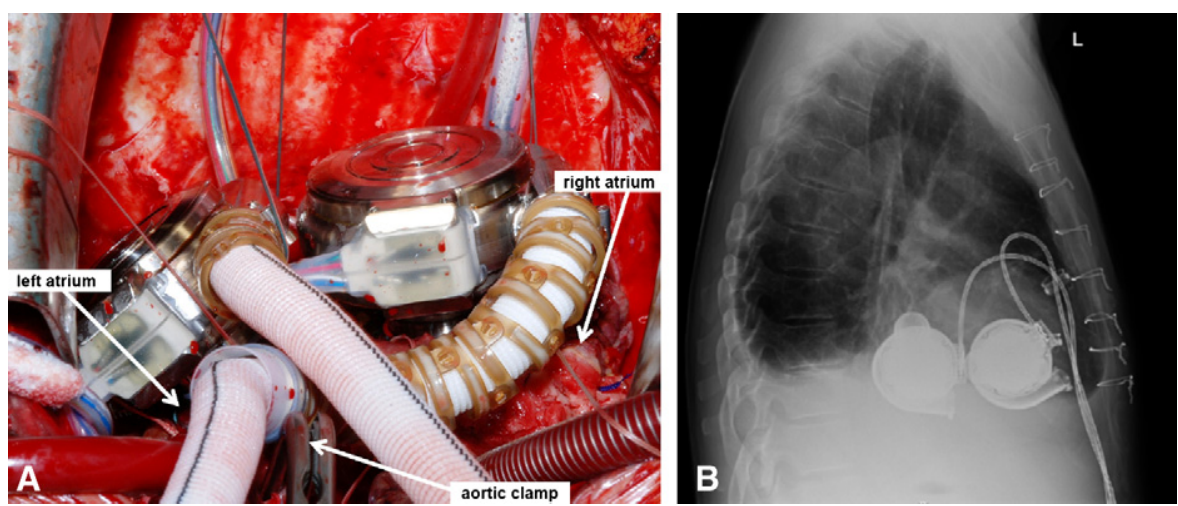

FIGURE 1. Positioning of the 2 HeartWare Ventricular Assist Device (HeartWare International, Inc, Framingham, Mass) centrifugal pumps as a total artificial heart. A, Intraoperative situs. B, Lateral chest radiograph.

sutures were sealed with biologic glue. Similar preparation followed on the ventricular aspect of the tricuspid annulus.

Two HVAD pumps were inserted in retrograde fashion through sewing rings into the left and right atria (Figure 1, $A$ and $B$ ). Outflow vascular grafts were anastomosed in an end-to-end fashion to the ascending aorta and pulmonary artery. Both drivelines were placed within the rectus muscle sheath toward the umbilicus and subcutaneously tunneled to the left and right upper quadrants. After deairing of both devices, pumps were started, and a left atrial pressure line was placed through the right upper pulmonary vein. Pump speeds were gradually increased during weaning from the extracorporeal circulation. Pump flow of $5.5 \mathrm{~L}$ on both sides was achieved, with the right pump running at $2600 \mathrm{rpm}$ and left pump running at $3000 \mathrm{rpm}$. Left atrial pressure remained stable at 6 to $10 \mathrm{~mm} \mathrm{Hg}$.
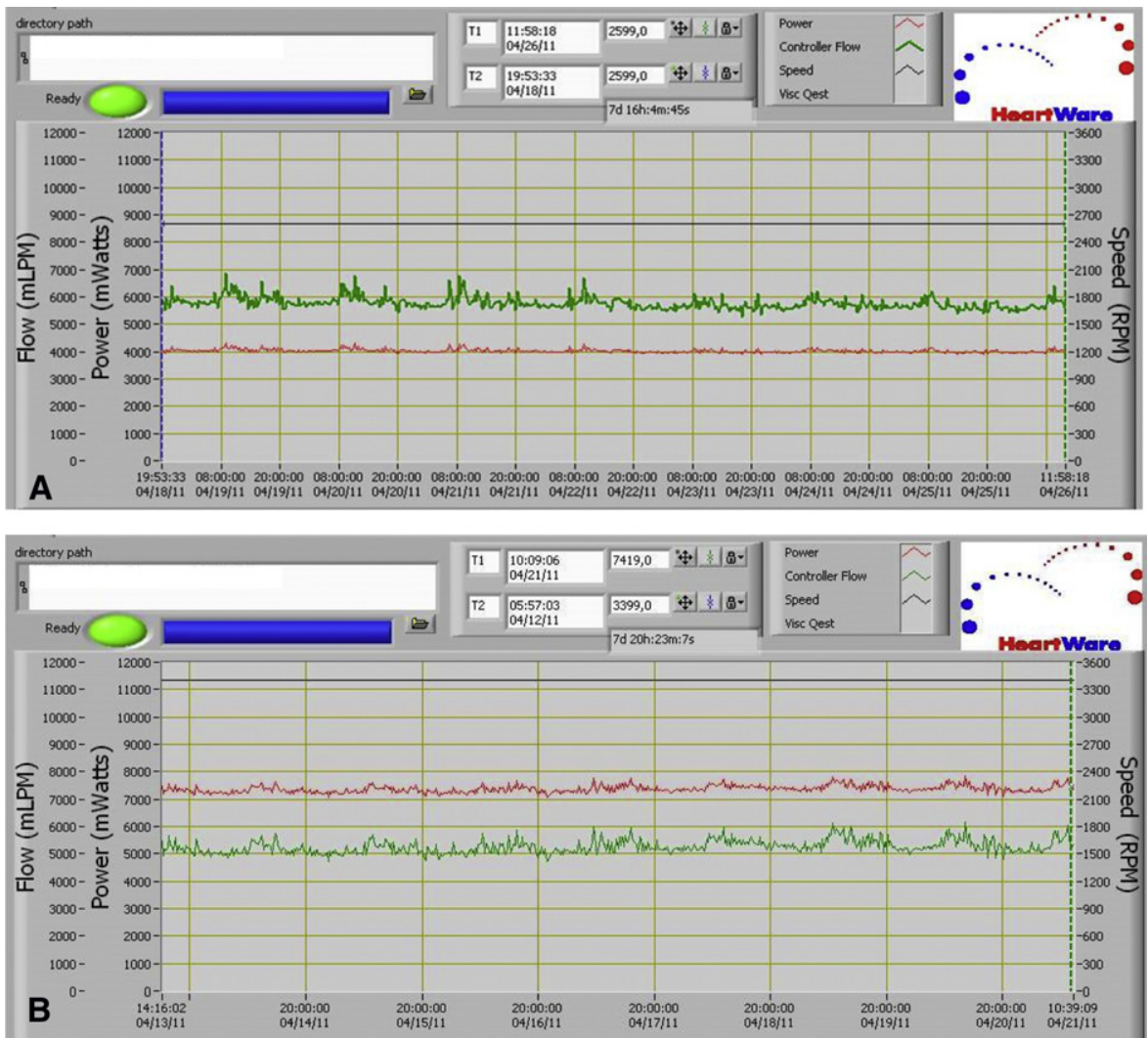

FIGURE 2. Log files of both HeartWare Ventricular Assist Device representing stable hemodynamic function of both pumps. A, Representative 7-day log file of the right ventricular assist device shows baseline speed of $2600 \mathrm{rpm}$, average power of $4 \mathrm{~W}$, and average flow of $5.8 \mathrm{~L} / \mathrm{min}$, indicating normal operation of the right ventricular assist device. B, Representative 7-day log file of the left ventricular assist device shows baseline speed of $3400 \mathrm{rpm}$, average power of $7.4 \mathrm{~W}$, and average flow of $5.3 \mathrm{~L} / \mathrm{min}$, indicating normal operation of the left ventricular assist device. 
A silicone breast implant $(225 \mathrm{~mL})$ was placed into the pericardial space to make room for later heart transplant. After closure of the chest, the patient was transferred to the ICU without any catecholamine support and with sufficient flow of both devices. The patient was successfully extubated on postoperative day 1 and remained in hemodynamically stable condition.

While ambulating on postoperative day 12 , the patient had an acute hemodynamic decompensation. The patient was transferred to the operating room for emergency operation. We found displacement of the silicone implant, pushing the right-sided pump into the right atrium and thereby kinking the outflow graft of the right device. Repositioning of the implant and the pump led to immediate hemodynamic stabilization. The patient was again extubated after a few hours, and the remaining stay at the hospital was completely uneventful (hemodynamics are shown in Figure 2, $A$ and $B$ ). Currently, the patient has successfully undergone cardiac transplant 14 weeks after implantation of both devices.

\section{DISCUSSION}

Our case substantiates the hypothesis that implantation of 2 HVAD centrifugal pumps may functionally serve as a total artificial heart. A silicone implant seems ideal to maintain intrapericardial space for subsequent heart transplantation; however, fixation within the pericardium is important. This option will continue to be important for patients with end-stage heart failure.

\section{References}

1. Strueber M, O’Driscoll G, Jansz P, Khaghani A, Levy WC, Wieselthaler GM, et al Multicenter evaluation of an intrapericardial left ventricular assist system. J Am Coll Cardiol. 2011;57:1375-82.

2. Wieselthaler GM, O Driscoll G, Jansz P, Khaghani A, Strueber M, HVAD Clinical Investigators. Initial clinical experience with a novel left ventricular assist device with a magnetically levitated rotor in a multi-institutional trial. J Heart Lung Transplant. 2010;29:1218-25.

3. Strueber M, Meyer AL, Malehsa D, Haverich A. Successful use of the HeartWare HVAD rotary blood pump for biventricular support. J Thorac Cardiovasc Surg. 2010;140:936-7.

4. Hetzer R, Krabatsch T, Stepanenko A, Potapov EV. Long-term biventricular support with the heartware implantable continuous flow pump. J Heart Lung Transplant. 2010;29:822-4.

\title{
Massive calcified left ventricular aneurysm and ventricular septum: Successful surgical repair and reconstruction
}

\author{
Xianqiang Wang, MD, Hengchao Wu, MD, and Hansong Sun, MD, Beijing, China
}

Massive calcification of a ventricular aneurysm, particularly of the ventricular septum, is uncommon after myocardial infarction. Surgical repair and reconstruction of an extensive calcified left ventricular aneurysm and septum are unrecorded to our knowledge. This report describes the successful surgical repair of a massive calcified left ventricular aneurysm and ventricular septum 13 years after the initial myocardial infarction.

\footnotetext{
From the Department of Cardiovascular Surgery, Cardiovascular Institute and Fuwai Hospital, Chinese Academy of Medical Sciences and Peking Union Medical College, Beijing, China.

Disclosures: Authors have nothing to disclose with regard to commercial support. Received for publication April 16, 2011; revisions received June 24, 2011; accepted for publication July 20, 2011; available ahead of print Sept 12, 2011.

Address for reprints: Hansong Sun, MD, Department of Cardiac Surgery; Cardiovascular institute and Fuwai Hospital, Chinese Academy of Medical Science and Peking Union Medical College, 167A Beilishi Road, Xi Chen District, Beijing 100037, People's Republic of China (E-mail: drsunhansong@sina.cn). J Thorac Cardiovasc Surg 2012;143:509-11

$0022-5223 / \$ 36.00$

Copyright (c) 2012 by The American Association for Thoracic Surgery doi:10.1016/j.jtcvs.2011.07.043
}

\section{CLINICAL SUMMARY}

A 63-year-old man presented with a 2-month history of indeterminate chest discomfort and increasing dyspnea. His medical history showed a notable extensive anterior infarction sustained 13 years previously. Electrocardiogram revealed significant abnormal Q-wave and ST-segment elevation in the anterior leads. A 2-dimensional echocardiogram showed a large apical aneurysm. The ventricular septum and apex became extensively calcified. There was a small cavity outside the apex that was filled with fluid, but it did not communicate with the cavity of the left ventricle. The left ventricle was significantly enlarged, but good left ventricular function was seen in the basal wall. Magnetic resonance imaging and cardiac catheterization confirmed these findings. Magnetic resonance imaging also showed a small thrombus on the aneurysmal inner wall. Coronary angiography showed that the left anterior descending and right coronary arteries were both occluded in their proximal portions.

The patient underwent operation, including resection of the left ventricular aneurysm and calcified septum, reconstruction of the ventricular septum, and a bypass to the right coronary artery. 